Research Article

\title{
Three-Dimensional Reconstruction Algorithm of Computed Tomography Imaging for Surgical Treatment and Rapid Rehabilitation Nursing of Renal Cell Carcinoma
}

\author{
Lijun Wang $\mathbb{D}$, Haiyan Lin $(\mathbb{D}$, Hong Zheng $(\mathbb{D}$, and Yuying Jiang $\mathbb{D}$ \\ Urinary Surgery, Taizhou Hospital of Zhejiang Province, Taizhou, Zhejiang 318050, China \\ Correspondence should be addressed to Yuying Jiang; wanglj8024@enzemed.com
}

Received 17 March 2021; Revised 19 April 2021; Accepted 2 May 2021; Published 10 May 2021

Academic Editor: Gustavo Ramirez

Copyright (c) 2021 Lijun Wang et al. This is an open access article distributed under the Creative Commons Attribution License, which permits unrestricted use, distribution, and reproduction in any medium, provided the original work is properly cited.

\begin{abstract}
This study was carried out to explore the promotion role of computed tomography (CT) imaging three-dimensional (3D) reconstruction technology and rapid rehabilitation nursing intervention (RRNI) in the treatment of patients with renal cell cancer (RCC) laparoscopic radical nephrectomy (LRN) in view of the patient's condition. 98 RCC patients who were admitted to the hospital from July 2019 to July 2020 were selected as the research subjects, and all patients underwent the LRN and the RRIN. Of which, 46 RCC patients were scanned with CT images (regarded as the CT group), and 46 RCC patients were scanned with CT images based on 3D reconstruction algorithms (regarded as the 3D CT group). The clinical efficacy and the life quality, pain degree, and adverse mood changes before and after the RRN were analyzed and compared. The results showed that the surgery time in the 3D CT group and the CT group was $130.2 \pm 42.8$ minutes and $162.4 \pm 38.5$ minutes, respectively $(P<0.05)$. The recurrence rate of RCC in both groups was $0 \%$. The estimated blood loss in the 3D CT group and the CT group was $93.6 \pm 35.5 \mathrm{~mL}$ and $90.3 \pm 40.2 \mathrm{~mL}$, respectively; the complication rate in the 3D CT and CT group was $5 \%$ and $12 \%$, respectively; the hospital stay in the 3D CT and CT group was $12.5 \pm 4.7$ days and $12.1 \pm 3.2$ days, respectively, which had no statistical significance $(P>0.05)$. The scores of visual analogue scale (VAS), 36-Item Short-Form Health Survey (SF-36), self-rating depression scale (SDS), and selfrating anxiety scale (SAS) of patients in the two groups were statistically significant $(P<0.05)$. It indicated that CT images based on the 3D reconstruction algorithm could be applied in LRN of RCC patients to shorten the surgery time and improve the surgical effect, and implementation of the RRN could relieve the adverse mood of RCC patients and effectively improve their life quality.
\end{abstract}

\section{Introduction}

As a kind of urinary system tumor, kidney tumor usually can be divided into benign and malignant tumor [1]. Of which, malignant tumor accounts for a relatively large proportion and can usually be divided into many types such as RCC, renal cell tumor, and renal metastasis [2]. RCC, also known as renal cancer, is the most common renal parenchymal malignant tumor. Patients suffering from RCC account for about $80 \%-90 \%$ of all renal malignancies. RCC is more often in people aged 50-60, most of who were male [3]. At present, the incidence and fatality rate of RCC all over the world are showing a significant increase trend. LRN is the first choice for the treatment of RCC, and it can also be applied to patients with clinical stage II and partial nephrectomy, so it is widely applied in the clinical treatment of RCC $[4,5]$. Currently, LRN has been widely recognized by more and more urologists and patients. LRN is superior to the open surgery in terms of shorter hospital stay and lower pain degree of patients after surgery, and it can reduce the financial pressure of the patient. LRN surgery can generally be divided into two approaches: transabdominal and retroperitoneal approach. The transabdominal approach has a larger abdominal operation space and a strong 3D effect, but it is easily interfered by abdominal organs. Although the operation space of the retroperitoneal approach is relatively small, it is not interfered by abdominal organs, is easy to expose, and has no risk of contamination. Thus, it is currently a clinical hot research topic [6]. LRN through the retroperitoneal approach can more intuitively and clearly 
show the renal artery so that it is easy for the surgery, but the operating space is relatively narrow, and the relationship among the adjacent organs is very close. Therefore, how to accurately locate and handle the renal pedicle vessels is of important significance for LRN through the retroperitoneal approach.

Imaging techniques for renal tumor diseases include ultrasound, CT, and magnetic resonance imaging (MRI) $[7,8]$. Ultrasound is usually only capable of preliminary diagnosis and qualitative analysis and is the main method of renal tumor screening. CT imaging can diagnose the benign and malignant kidney tumors and can also determine its clinical stage. MRI is similar to CT in terms of diagnostic functions. With the development of medical imaging technology and the concept of "precision surgery," twodimensional images can no longer meet the current requirements. In recent years, 3D reconstructed images drawn by the built-in software of CT instruments have shown more and more advantages and can show the $3 \mathrm{D}$ anatomical structures of kidney tumors and renal pedicles clearly. Wake et al. found that cognitive renal tumors based on CT and MRI data were poorly localized, and 3D reconstruction models could improve the accuracy of tumor localization and may provide assistance through appropriate surgical methods [9]. Therefore, the feasibility and prospects of $3 \mathrm{D}$ CT reconstruction imaging technology in the diagnosis and treatment of renal tumors are very promising. RRNI is a comprehensive nursing technology that combines multiple disciplines such as nursing and nutrition. It can adopt a series of optimized measures during the perioperative period with evidence-based medical evidence. In addition, it can reduce or block the stress response of patients during the perioperative period through the multidisciplinary cooperation of surgery, anesthesia, nursing, and nutrition. Thus, it can promote the recovery of surgical patients as soon as possible and fully improve the postoperative physiological state. Therefore, it has already played an important role in the surgical treatment of colorectal cancer, esophageal cancer, and other diseases [10].

Based on the advantages of the $3 \mathrm{D}$ reconstruction algorithm, 3D reconstruction technology was applied for $3 \mathrm{D}$ reconstruction of the patient's CT image data and utilized to the LRN and RRN of RCC patients to explore the clinical efficacy and the life quality, pain degree, and adverse mood of the patients before and after the nursing. This study was to provide an important basis for the optimal selection of clinical treatment and surgical plans and subsequent nursing interventions for RCC patients.

\section{Materials and Methods}

2.1. Research Objects. 98 RCC patients who were admitted to hospital from July 2019 to July 2020 were selected and treated with LRN. Among them, 46 patients were scanned with CT images (CT group), and 46 patients were scanned with CT images based on $3 \mathrm{D}$ reconstruction algorithms (3D CT group). There were 30 males and 16 females in the CT group and 28 males and 18 females in the 3D CT group. This experiment had been approved by the Ethics Committee of the Hospital, and all the patients included had understood the experiment fully and signed informed consent.

The inclusion criteria were defined as below: patients performed with CT examination; patients who were diagnosed as RCC by pathological results; patients without abnormal heart or lung function; patients without history of abdominal surgery; and patients with normal blood coagulation function. The exclusion criteria could be determined as follows: patients who were contraindicated in LRN; patients who were allergic to contrast agents; and patients with underlying diseases related to LRN.

2.2. CT Image Scanning Based on 3D Reconstruction Algorithm. The CT contrast agent referred to an isotonic contrast agent iodixanol. The patients were required to fast for 4 hours before the examination. In order to avoid artifacts caused by breathing motion to interfere with the image, the patients were allowed to drink $500-1,000 \mathrm{~mL}$ of water half an hour before the examination and $500 \mathrm{~mL}$ of water again before the CT examination. The tube voltage was set to $120 \mathrm{kV}$, the tube current was set to $300 \mathrm{~mA}$, the layer thickness was $5 \mathrm{~mm}$, and the pitch was 0.984 . The patient was in the supine position and performed a CT scan from the level of the xiphoid process to the level of the symphysis pubis.

The preoperative planning and intraoperative guidance of the CT group were realized by using the two-dimensional CT scan images and the computed tomography angiography (CTA) and computed tomography urography (CTU) equipped for the instrument. Preoperative planning of the 3 D CT group was realized using the Mimics 17.0 software for CT image segmentation and $3 \mathrm{D}$ reconstruction, and a $3 \mathrm{D}$ reconstruction model of the kidney was constructed to realize the simulated positioning of renal pedicle vessels, and the possible intraoperative damage or vascular leakage was predictively analyzed. In addition, the intraoperative guidance was realized through $3 \mathrm{D}$ reconstruction models and artificial fusion images for positioning guidance. The CT images were segmented with the threshold segmentation algorithm (TSA) and region growing algorithm (RGA). The $3 \mathrm{D}$ reconstruction algorithm of $\mathrm{CT}$ image based on the Mimics platform is for image reconstruction. The Mimics software was used to generate and edit the $3 \mathrm{D}$ images. Data of all kinds of equipment such as CT and MRI could be imported. The 3D model was constructed with the image segmentation and registration fusion technology. After modification, the imported data could be outputted in computer-aided design (CAD) or rapid prototyping (RP) format.

2.3. Quality Assessment of the 3D Reconstructed CT Image. Two urologists and radiographers with more than 5 years of experience were selected to evaluate the quality of $3 \mathrm{D}$ reconstructed CT images. The scoring standards are shown in Table 1. 
TABLE 1: Quality assessment of 3D reconstructed CT image.

\begin{tabular}{|c|c|c|c|c|}
\hline Score & Trunk and branches of renal artery/renal vein & Edge of blood vessel & $\begin{array}{l}\text { Artifacts caused by breathing } \\
\text { motion }\end{array}$ & Other \\
\hline 1 & The trunk and primary branches could be displayed clearly & Fuzzy & Yes or no & - \\
\hline 2 & $\begin{array}{c}\text { The trunk, primary, and secondary branches could be } \\
\text { displayed clearly }\end{array}$ & Smooth & No & $\begin{array}{l}\text { The secondary } \\
\text { branches }<4\end{array}$ \\
\hline 3 & $\begin{array}{c}\text { The trunk, primary, secondary, and tertiary branches could } \\
\text { be displayed clearly }\end{array}$ & Smooth & No & $\begin{array}{l}\text { The secondary } \\
\text { branches }<5\end{array}$ \\
\hline 4 & $\begin{array}{c}\text { The trunk, primary, secondary, and tertiary branches could } \\
\text { be displayed clearly }\end{array}$ & Smooth & No & $\begin{array}{l}\text { The secondary } \\
\text { branches } \geq 5\end{array}$ \\
\hline 5 & $\begin{array}{c}\text { The trunk, primary, secondary, tertiary, and quaternary } \\
\text { branches could be displayed clearly }\end{array}$ & Smooth & No & - \\
\hline
\end{tabular}

2.4. LRN Treatment. LRN was performed through a retroperitoneal approach. The patient was preoperatively waterfree and fasted, the enema and the skin were cleaned, urinary catheters were indwelled, and endotracheal intubation was performed under general anesthesia. The patient was placed in a lateral position with raised waist bridge, lowered head, and lower limbs and then disinfected. The incision (approximately $2 \mathrm{~cm}$ ) was cut at the edge of the 12th rib of the posterior axillary line, and the muscle and the posterior peritoneal space were separated. A glove air bag containing $700 \mathrm{~mL}$ of air was placed and retained for 180 seconds, and then the air was exhausted and the air bag was taken out. A transverse incision $(1.5 \mathrm{~cm}$ in size) was cut above the midaxillary iliac crest; the index finger was allowed to deepen into the posterior abdominal cavity from the incision to insert the $12 \mathrm{~mm}$ trocar carefully. The anterior axillary line was cut below the costal margin for approximately $0.5 \mathrm{~cm}$, a $5 \mathrm{~mm}$ trocar was inserted in the same way as the previous operation, and then a $12 \mathrm{~mm}$ trocar was placed below the edge. The pneumoperitoneum was slowly established, and a laparoscope was placed. After the retroperitoneal fat was removed, blunt separation was performed in the prerenal fascia, posterior fascia, and psoas major with an ultrasonic knife. Then, the renal artery and renal vein were separated. The ligation and separation were realized with the Hem-olok to free the ureter. The pararenal space was enlarged, the kidneys and fat sacs were cut, and the bleeding was stopped through electrocoagulation with a PK cutter. The drainage tube was placed to a depth of $20 \mathrm{~cm}$ and drawn out from the incision at the midaxillary iliac spine, and the trocar was taken out. Finally, the incision was sutured to finish the surgery.

2.5. Rapid Rehabilitation Nursing Intervention. RRN was for intervention treatment. The pain and life quality of patient during hospitalization were evaluated using the VAS score [11] and the SF-36 scale [12], and changes in the adverse mood were evaluated using the SDS scale [13] and SAS scale [14].

The RRN was realized by the following modes before, during, and after the LRN. Before LRN, health education was given to patient after admission, so that he could fully understand the disease and nursing implementation plan and actively cooperate with medical staff; the emotional state of the patient was closely focused on to help the patient establish a good state of mind; the patient was informed of preoperative dietary precautions, which included fasting for 6 hours and no drinking water for 2 hours; in addition, the patient was required to take $800 \mathrm{~mL}$ of $10 \%$ glucose solution before 8 o'clock in the evening before surgery. During LRN, the emotional state of the patient was concerned, and positive comfort was given; the temperature in the operating room was controlled reasonably at $20-25^{\circ} \mathrm{C}$; corresponding measures could be taken to heat the infusion and washing solution if necessary; and mattresses could also be adopted to keep warm and ensure good insulation measures for the patient. After LRN, the changes in the psychological state of patient was closely concerned, and necessary psychological counseling could be provided according to the actual situation; the patient was supervised to take medications on time and given with detailed medication guidance, and dietary guidance, including warm water, was allowed 6 hours after surgery and a small amount of liquid food could be taken after 1 day, and then semiliquid food and normal diet could be allowed according to the recovery situation; the families were supervised to help move the patient's limbs, help the patient on bed exercises such as deep breathing, and try to get out of bed according to the situation the next day; the state of the patient's urine was observed, the urine bag was changed frequently, and the drainage tube was removed as soon as possible; the patient was informed of the principle of daily diet and avoiding strenuous exercise before leaving the hospital, and appropriate medication guidance was provided; and the patient's condition was asked for promptly and the timely follow-up visit was guaranteed.

2.6. Statistical Analysis. SPSS 20.0 statistical software was employed for analysis. Measurement data were indicated as mean \pm standard deviation ( $x \pm s$ ), and the count data were expressed as percentage (\%). The two groups of patients were compared by $t$-test in gender, age, body mass index (BMI), American Society of Anesthesiologists (ASA) score, tumor location, tumor maximum diameter, accessory renal artery, and hemoglobin. The operation time, intraoperative blood loss, hospital stay, follow-up time, blood transfusion rate, complication rate, and tumor recurrence rate were 
compared between the two groups by one-way analysis of variance. The $t$-test or $\chi^{2}$ test was adopted. If $P<0.05$, the difference was determined as statistically meaningful.

\section{Results}

3.1. Analysis of 3D Reconstruction Effect of the CT Image. The image reconstruction for patients in 3D CT group was realized by $3 \mathrm{D}$ reconstruction algorithm of CT image based on the Mimics platform, so that the tumor location and depth, structure characteristics of renal pedicle vascular, and variant anatomy could be shown clearly and intuitively (Figure 1). The image $3 \mathrm{D}$ reconstruction, preoperative planning, and intraoperative guidance were realized successfully through the Mimics 17.0 software in the 3D CT group. For the patients in CT group, the CT scan data was utilized for surgical planning and intraoperative guidance, and the LRN surgery was also successful.

In the 3D CT group, the 3D CT image of the renal pedicle vessels of 48 RCC patients were assessed, and the results are shown in Table 2 . There were 3 cases in 1 score, 28 cases in 2 scores, and 17 cases in 3 scores among the renal vein images; and there were 4 cases with 2 scores, 4 cases with 3 scores, 30 cases with 4 scores, and 10 cases with 5 scores among the renal artery. A total of 46 cases of $3 \mathrm{D}$ image models were effectively and completely evaluated. There was 1 image with incomplete completeness of the model due to insufficient contrast agent, but the characteristics of the renal vein/arterial trunk were still clearly displayed, so the surgery was not affected accordingly. In summary, the effectiveness of reconstructed $\mathrm{CT}$ images based on $3 \mathrm{D}$ reconstruction algorithms reached $95.9 \%$.

3.2. Clinical Information of Patients with Renal Cell Carcinoma. The differences between the two groups of patients in gender, age, BMI, ASA score, tumor location, tumor maximum diameter, accessory renal artery, and hemoglobin were not statistically meaningful $(P<0.05)$, as shown in Table 3.

3.3. Analysis of Clinical Efficacy. The average surgery time of patients in the 3D CT group was $130.2 \pm 42.8$ minutes, which was obviously shorter than that of the CT group $(162.4 \pm 38.5$ minutes $)(P<0.05)$; the estimated blood loss of patients in the 3D CT group and the CT group was not greatly different, which was $93.6 \pm 35.5 \mathrm{~mL}$ and $90.3 \pm 40.2 \mathrm{~mL}$, respectively $(P>0.05)$, as shown in Figure 2.

The hospital stays and follow-up time between the two groups were compared and analyzed, and the results are shown in Figure 3. It revealed that the hospital stay (12.5 \pm 4.7 days and $12.1 \pm 3.2$ days, respectively) and the follow-up time $(4.6 \pm 2.5$ months and $5.8 \pm 2.7$ months, resp.) in the $3 \mathrm{D}$ CT group and the CT group had no statistically meaningful difference $(P>0.05)$.

The pathological results of both groups were determined as RCC both. In the $3 \mathrm{D}$ CT group, the intraoperative blood transfusion rate was $16 \%$, the complication rate was $5 \%$, and the tumor recurrence rate was $0 \%$, and the above indicators in the CT group was $21 \%, 12 \%$, and $0 \%$, respectively. There was no observable difference in the blood transfusion rate, complication rate, and tumor recurrence rate between the two groups $(P>0.05)$, as shown in Figure 4.

There was no statistical difference between 3D CT group and CT group in terms of postoperative hemoglobin $(114.56 \pm 13.28 \mathrm{~g} / \mathrm{L}$ vs. $108.63 \pm 16.38 \mathrm{~g} / \mathrm{L})$ and hemoglobin change $(15.73 \pm 10.57 \mathrm{~g} / \mathrm{L}$ vs. $18.37 \pm 14.32 \mathrm{~g} / \mathrm{L})(P>0.05)$, as illustrated in Figure 5.

3.4. Nursing Intervention Effect. As shown in Figure 6, the patient's VAS score and SF-36 score before intervention was $3.91 \pm 0.45$ points and $92.54 \pm 9.43$ points, respectively; and they were $2.21 \pm 0.32$ points and $110.54 \pm 10.53$ points, respectively, after intervention. Among them, the VAS score of patients after intervention was obviously lower than that before intervention, while the SF-36 score after intervention was much greater than the score before intervention, and the difference was statistically significant $(P<0.05)$.

Figure 7 reveals that the SDS score and SAS score of patients before and after the intervention were $58.46 \pm 8.51$ scores vs. $43.08 \pm 8.13$ scores and $54.97 \pm 10.31$ scores vs. $47.82 \pm 7.92$ scores, respectively $(P<0.05)$.

\section{Discussion}

The probability of renal pedicle vascular mutation in the kidney of patients with malignant tumors is generally higher than that of the normal population, which increases the complexity of the surgery to a certain extent. The key part of LRN is the precise positioning and treatment of renal pedicle vessels. Studies have shown that the $3 \mathrm{D}$ reconstruction model of the kidney can accurately and objectively display the renal arteries and veins as well as other tissue structures clearly [15]. Huang et al. used the Urmedix-3D system to reconstruct the 3D CT structure of human kidneys and renal lesions. The results showed that the reconstructed 3D model can objectively evaluate the spatial anatomical relationship of the lesions, thereby providing help for surgical planning [16]. This article uses a $3 \mathrm{D}$ reconstruction algorithm to process CT images, which can clearly see the patient's tumor location and depth, and renal pedicle vascular structure characteristics, indicating that this algorithm can improve the accuracy of CT image detection results and help identify renal pedicle vascular information. This provided an information reference for doctors to develop personalized treatment plans for patients and even follow-up LRN operations.

In this paper, the operation time of 3D CT group was $130.2 \pm 42.8(\mathrm{~min})$, which was less than $162.4 \pm 38.5(\mathrm{~min})$ of CT group, $P<0.05$; the complication rate of $3 \mathrm{D}$ CT group was $5 \%$ and that of CT group was $12 \%$. It shows that $3 \mathrm{D}$ CT reconstruction images can shorten the operation time and improve the surgical effect in LRN surgical treatment. Hu et al. found that $3 \mathrm{D}$ spatial CT measurement of anatomical structures can help improve the accuracy of LPN performance and reduce the warm ischemia time compared with 

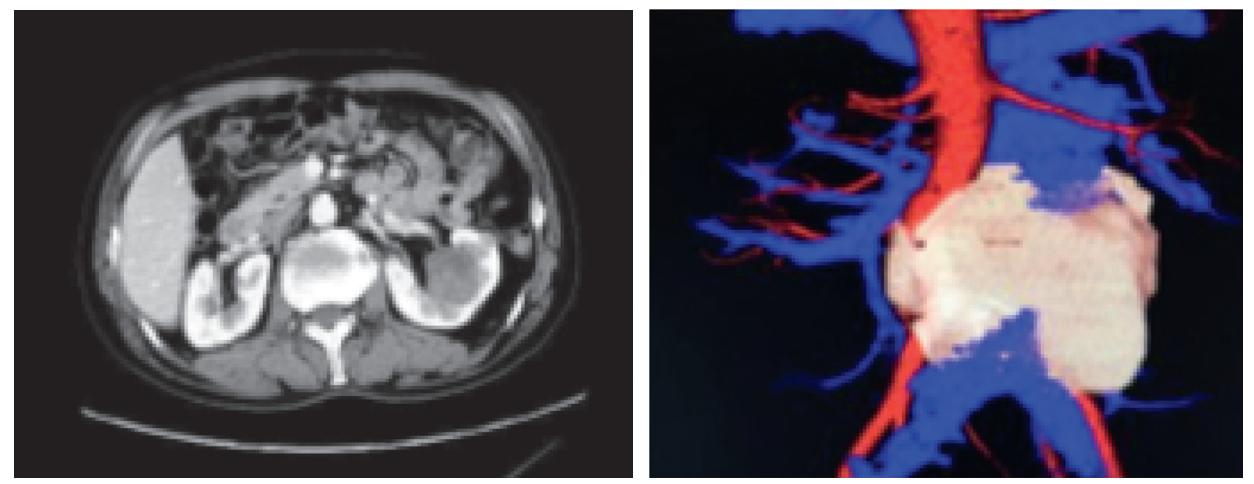

(a)
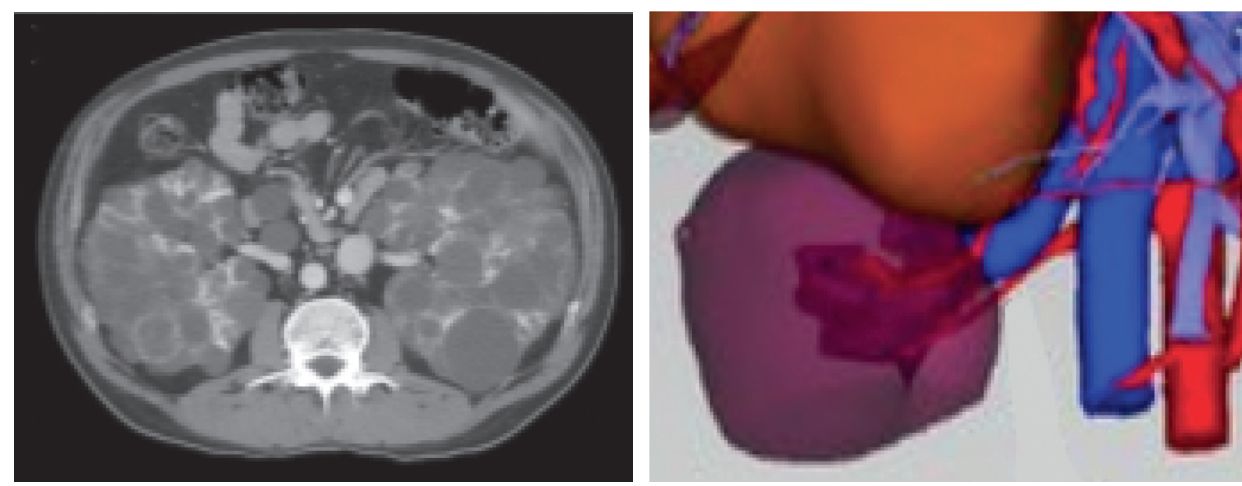

(b)

FIGURE 1: CT image based on 3D reconstruction algorithm. (a) The variation of renal pedicle blood vessel. (b) The variation and deformation of renal vein branch caused by compression of the renal tumor.

TABLE 2: Assessment results of quality of 3D CT images.

\begin{tabular}{lcc}
\hline Score & 3D CT of renal vein & 3D CT of renal artery \\
\hline 1 & 3 & 0 \\
2 & 28 & 4 \\
3 & 17 & 4 \\
4 & 0 & 30 \\
5 & 0 & 10 \\
\hline
\end{tabular}

TABLE 3: Comparison of clinical information of patients with RCC.

\begin{tabular}{lccc}
\hline & CT $(n=48)$ & 3D CT $(n=48)$ & $P$ \\
\hline Gender (male/female) & $28 / 18$ & $30 / 16$ & 0.765 \\
Age (years old) & $52.98 \pm 10.38$ & $53.25 \pm 11.86$ & 0.729 \\
BMI $\left(\mathrm{kg} \mathrm{m}^{-2}\right)$ & $21.87 \pm 1.76$ & $21.62 \pm 3.28$ & 0.976 \\
ASA score & $1.23 \pm 0.58$ & $1.26 \pm 0.46$ & 0.723 \\
$\begin{array}{l}\text { Tumor location (left/ } \\
\text { right) }\end{array}$ & $20 / 28$ & $22 / 26$ & 0.741 \\
$\begin{array}{l}\text { Tumor maximum } \\
\text { diameter (cm) }\end{array}$ & $6.23 \pm 2.87$ & $5.26 \pm 1.35$ & 0.187 \\
$\begin{array}{l}\text { Accessory renal artery } \\
\text { (branch) }\end{array}$ & $0.3 \pm 0.4$ & $0.5 \pm 0.4$ & 0.214 \\
Hemoglobin $\left(\mathrm{g} \mathrm{L}^{-1}\right)$ & $126.38 \pm 20.39$ & $129.37 \pm 17.36$ & 0.527 \\
\hline
\end{tabular}

traditional CT measurement [17], which was similar to the results of this study; the difference between this study and $\mathrm{Hu}$ et al.'s lied in that there was an obvious difference in warm ischemia time $(26.7 \pm 6.4$ vs. $31.9 \pm 7.0$ minutes $)$ and tumor resection time $(8.4 \pm 2.6$ vs. $10.4 \pm 2.8$ minutes $)$, but

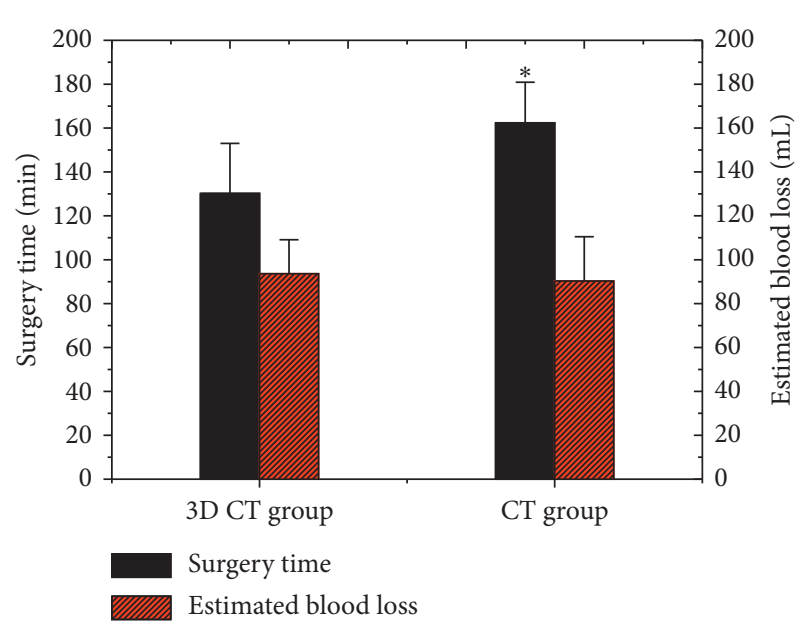

Figure 2: Comparison of average surgery time and estimated blood loss of two groups (note: ${ }^{*}$ indicates $P<0.05$ in contrast to the $3 \mathrm{D}$ CT group).

there was no considerable difference in surgery time between the CT and 3D CT group, which maybe because that research object of Hu et al. was LNP patients diagnosed with T1 renal mass, which was different from the research object of this study. Wang et al. [18] compared the effectiveness and safety of routine CT examination and individualized $3 \mathrm{D}$ visualization technology (3D VT) in the surgical planning and perioperative effects of LPN treatment for RCC and found 


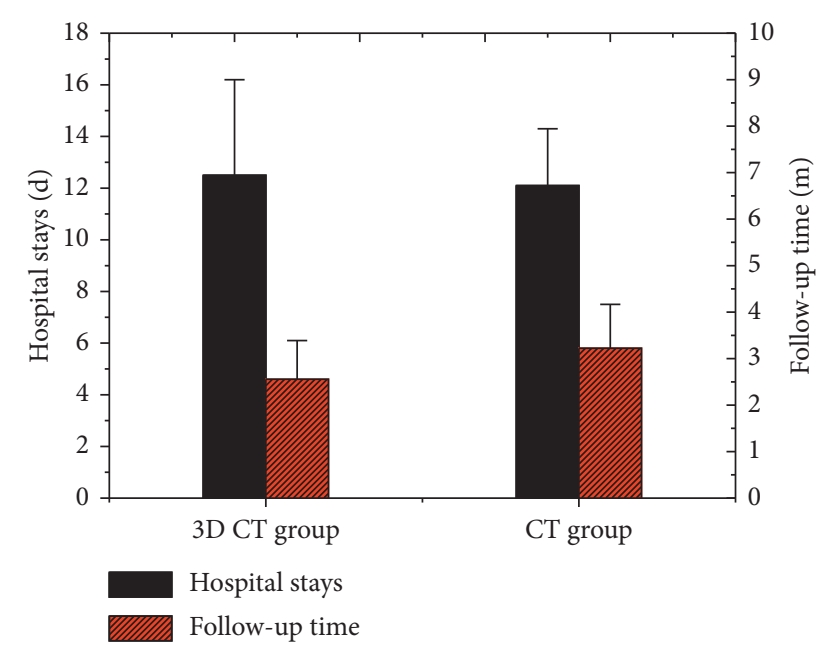

FIGURE 3: Comparison of in-hospital stay and follow-up time in the two groups.

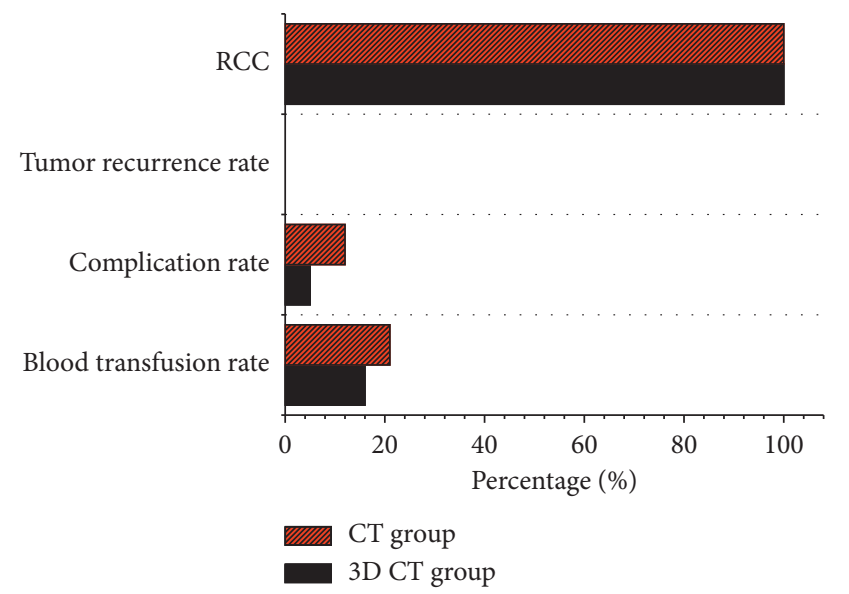

FIgURE 4: Comparison of blood transfusion rate, complication rate, tumor recurrence rate, and RCC of patients in the two groups.

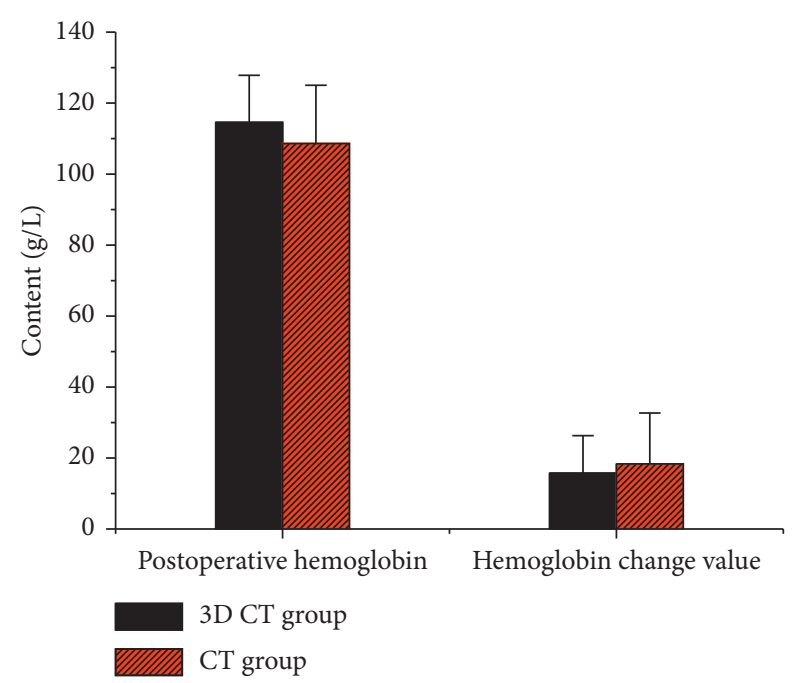

FIGURE 5: Comparison of postoperative hemoglobin and hemoglobin change.

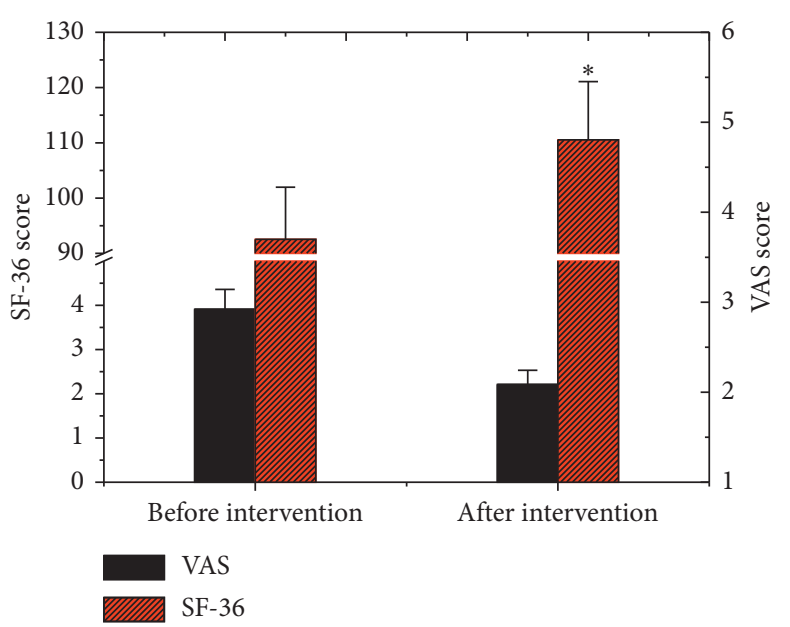

FIgURE 6: Scores of visual analogue scale and SF-36 before and after intervention (note: * suggests $P<0.05$ in contrast to the scores before intervention).

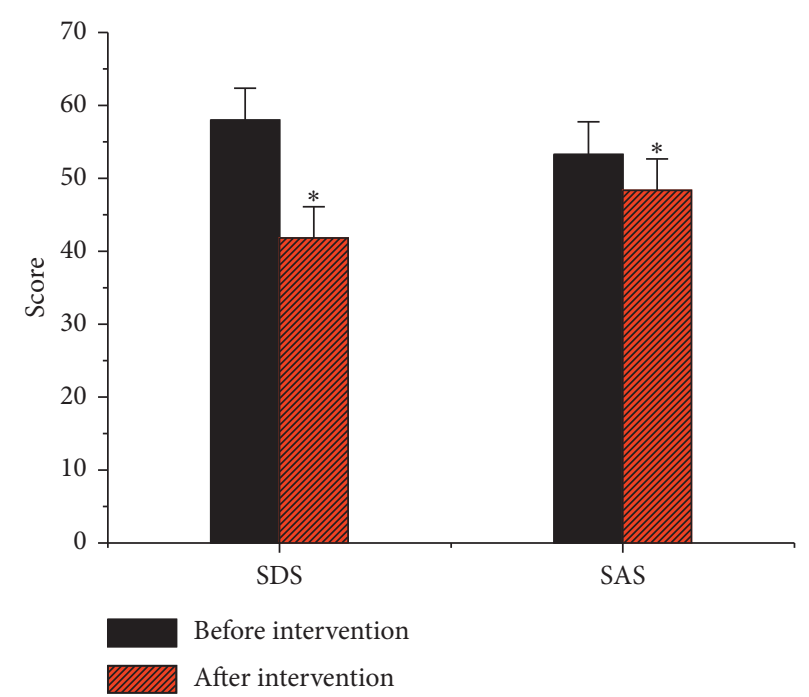

Figure 7: Changes in mood change before and after the intervention (note: ${ }^{*}$ indicates $P<0.05$ in contrast to the score before intervention).

that the surgery time $(126.7 \pm 36.4$ vs. $154.8 \pm 34.7$, $P=0.018)$ and the incidence of postoperative urine leakage ( $0 \%$ vs. $22.2 \%, P=0.033$ ) were greatly reduced in the $3 \mathrm{D}$ VT group when RENAL score of the tumor was $\geq 8$, which was consistent with the results in this experiment. Due to the lack of medical knowledge, some patients generally have fear or anxiety before surgery. The RRN was adopted to give full play to the principle of patient-centered treatment, provide a variety of psychological care and health education, and enhance the patient's confidence in treatment [19]. The SDS and SAS scores of RCC patients after nursing were improved obviously, indicating that RRN could improve the adverse mood of LRN patients. The improvement in VAS and SF-36 scores of RCC patients in this study indicated that RRN played a good role in the prevention and management of pain after LRN, was also conducive to improving the life 
quality of patients, and had positive significance for subsequent rehabilitation.

\section{Conclusion}

In this study, 98 RCC patients were divided into a CT group (two-dimensional CT image) and a 3D CT group (3D CT image reconstruction), and all received the RRNI. The results showed that 3D CT reconstruction images greatly shortened the surgery time and improved the surgical effect in the LRN of RCC patients. RRN could alleviate the adverse mood of patient and effectively improve the life quality. However, the number of samples in this study was limited, and individual patient differences had greater impacts on the surgical process and results. In the follow-up, it was necessary to increase the number of samples and increase the analysis of other intraoperative or postoperative observation indicators and further explore the impacts of CT imaging technology based on $3 \mathrm{D}$ reconstruction algorithms on the efficacy of LRN in patients with RCC. In short, the results of this study could provide a reliably theoretical basis for improving the efficacy of LRN for patients with RCC and for the research on nursing intervention.

\section{Data Availability}

No data were used to support this study.

\section{Conflicts of Interest}

The authors declare that they have no conflicts of interest regarding the publication of this paper.

\section{References}

[1] A. K. Lalwani, S. M. Faiq, and J. Ahuja, "Solitary fibrous tumor of kidney," Journal of the College of Physicians and Surgeons-Pakistan: JCPSP, vol. 28, no. 7, pp. 564-565, 2018.

[2] N. Petejova and A. Martinek, "Renal cell carcinoma: review of etiology, pathophysiology and risk factors," Biomedical $\mathrm{Pa}$ pers, vol. 160, no. 2, pp. 183-194, 2016.

[3] H. I. Wettersten, O. A. Aboud, P. N. Lara Jr, and R. H. Weiss, "Metabolic reprogramming in clear cell renal cell carcinoma," Nature Reviews Nephrology, vol. 13, no. 7, pp. 410-419, 2017.

[4] F. Kunath, S. Schmidt, L. M. Krabbe et al., "Partial nephrectomy versus radical nephrectomy for clinical localised renal masses," The Cochrane Database of Systematic Reviews, vol. 5, no. 5, Article ID CD012045, 2017.

[5] M. C. Mir, I. Derweesh, F. Porpiglia, H. Zargar, A. Mottrie, and R. Autorino, "Partial nephrectomy versus radical nephrectomy for clinical T1b and T2 renal tumors: a systematic review and meta-analysis of comparative studies," European Urology, vol. 71, no. 4, pp. 606-617, 2017.

[6] B. Yuan, Y. Wang, J. Gao, Y. Zhang, Y. Fu, and W. An, "Lower pole approach in retroperitoneal laparoscopic radical nephrectomy: a new approach for the management of renal vascular pedicle," World Journal of Surgical Oncology, vol. 16, no. 1 , p. $31,2018$.

[7] G. Kiss, D. Korda, G. Szabó et al., "Oncological screening of kidney transplant patients: the role of ultrasound examination," Transplantation Proceedings, vol. 51, no. 4, pp. 1231-1233, 2019.
[8] Z. Xie, G. Zhu, L. Cheng, J. Liu, H. Ye, and H. Wang, "Solitary fibrous tumor of the kidney: magnetic resonance imaging characteristics in 4 patients," Medicine (Baltimore), vol. 97, no. 34, Article ID e11911, 2018.

[9] N. Wake, J. S. Wysock, M. A. Bjurlin, H. Chandarana, and W. C. Huang, "“Pin the tumor on the kidney:" an evaluation of how surgeons translate CT and MRI data to $3 \mathrm{D}$ models," Urology, vol. 131, pp. 255-261, 2019.

[10] F. Xu, P. Yu, and L. Li, "Rapid rehabilitation nursing in postoperative patients with colorectal cancer and quality of life," Oncology Letters, vol. 18, no. 1, pp. 651-658, 2019.

[11] G. M. Kim, J. Y. Won, M. D. Kim et al., "Cryoablation of hepatocellular carcinoma with high-risk for percutaneous ablation: safety and efficacy," CardioVascular and Interventional Radiology, vol. 39, no. 10, pp. 1447-1454, 2016.

[12] L. Sandbergen, A. S. Spriensma, J. J. de la Rosette, and M. P. Laguna, "Health-related quality of life in localized renal masses: a matter of sparing nephrons or minimizing the incision?" Urologic Oncology, vol. 38, no. 2, pp. 43.e1-43.e11, 2020.

[13] W. Wang, G. Lou, and Y. Zhang, "Olanzapine with ondansetron and dexamethasone for the prevention of cisplatinbased chemotherapy-induced nausea and vomiting in lung cancer," Medicine (Baltimore), vol. 97, no. 37, Article ID e12331, 2018.

[14] X. Sheng, F. Liu, J. Zhou, and R. Liao, "Psychological status and sleep quality of nursing interns during the outbreak of COVID-19," Nan Fang Yi Ke Da Xue Xue Bao, vol. 40, no. 3, pp. 346-350, 2020.

[15] K. M. Jones, L. B. Solnes, S. P. Rowe et al., "Use of quantitative SPECT/CT reconstruction in $99 \mathrm{mTc}$-sestamibi imaging of patients with renal masses," Annals of Nuclear Medicine, vol. 32, no. 2, pp. 87-93, 2018.

[16] J. Huang, S. Lü, Z. Hu, C. Huang, Y. Li, and Q. Wei, “Threedimensional reconstruction of human kidney based on UroMedix-3 D system and its application in kidney surgery," Nan Fang Yi Ke Da Xue Xue Bao, vol. 39, no. 5, pp. 614-620, 2019.

[17] Z. F. Hu, S. D. Lv, J. F. Huang et al., "Three-dimensional spatial measurement versus conventional CT planning in laparoscopic partial nephrectomy for renal tumors," Nan Fang Yi Ke Da Xue Xue Bao, vol. 38, no. 5, pp. 606-611, 2018.

[18] Z. Wang, L. Qi, P. Yuan et al., "Application of three-dimensional visualization technology in laparoscopic partial nephrectomy of renal tumor: a comparative study," Journal of Laparoendoscopic \& Advanced Surgical Techniques, vol. 27, no. 5, pp. 516-523, 2017.

[19] A. Tam, S. Mac, W. Isaranuwatchai, and M. Bayley, "Costeffectiveness of a high-intensity rapid access outpatient stroke rehabilitation program," International Journal of Rehabilitation Research, vol. 42, no. 1, pp. 56-62, 2019. 